\title{
Surface mobility of Ag on Pd(100) measured by specular helium scattering
}

\author{
C. Félix, G. Vandoni, W. Harbich, J. Buttet, and R. Monot \\ Institut de Physique Expérimentale, Ecole Polytechnique Fédérale, 1015 Lausanne, Switzerland
}

(Received 19 December 1995)

\begin{abstract}
We study the deposition and the very first steps of nucleation and growth of $\mathrm{Ag}$ on $\mathrm{Pd}(100)$ with thermal energy atom scattering. This technique is a very sensitive and nonperturbing probe to surface point defects, which permits an in situ and in-time monitoring of the deposition. The intention of this paper is to give a detailed description of the approach used in our work. The form of the specularly reflected helium signal as a function of coverage and surface temperature is compared to a theoretical curve, which is computed by solving a system of rate equations that describe the formation and destruction of clusters during the deposition process. The analysis of the experimental data gives two main results. The diffusion parameters (activation barrier $E_{d}=0.37 \pm 0.03 \mathrm{eV}$ and preexponential factor $\nu_{0}=8 \times 10^{9} \mathrm{~s}^{-1}$ ) have been extracted for the system $\mathrm{Ag}$ on $\operatorname{Pd}(100)$. We find furthermore that all silver atoms impinging on a zone of $6.1 \AA$ around an adatom on the surface are captured by it at surface temperatures well below the onset of thermally activated mobility. The origin of this phenomenon is discussed and tentatively assigned to a combined effect of transient and neighbor driven mobility. [S0163-1829(96)04747-9]
\end{abstract}

\section{INTRODUCTION}

The study of the early stages of epitaxial growth is a field of considerable interest because of the fundamental phenomena that are implied as well as the importance of related applications, such as microelectronics or catalysis. ${ }^{1,2}$ The structure of the small particles grown on an atomically flat surface depends strongly on the mobility of the adatoms or the small particles of the deposited material.

At least two phenomena can influence the early stages of growth: deposition and thermal diffusion. The study of the deposition process raises the controversial question of the transient mobility. An atom evaporated from a Knudsen cell has a thermal energy of the order of $0.16 \mathrm{eV}$, which has to be summed to the binding energy (about $3 \mathrm{eV}$ ), which it gains by adsorption on the surface. This sum is high compared to typical diffusion barriers of metal on metal systems. ${ }^{3,4}$ The deposited atoms have to dissipate all this energy to thermalize on the surface. The question is how quickly does this happen and what is its influence on the mobility? This question becomes less important as soon as thermal mobility becomes high. At this point the structure is governed by nucleation and growth, which can be modeled with rate equations.

In the case of a metal deposited on a metallic surface, numerous experimental and theoretical studies have been performed to determine the parameters of the diffusion mechanisms. Extensive review articles have been published concerning the diffusion of metal atoms on metal surfaces and the various experimental techniques ${ }^{4-6}$. Among these techniques are the direct visualization methods such as field ion microscopy (FIM) ${ }^{7-9}$ scanning transmission electron microscopy (STEM), ${ }^{10}$ or scanning tunneling microscopy (STM). ${ }^{11,12}$ While the first method visualizes simple atomic hops, the analysis of STEM and STM micrographs relates the island density to the surface temperature and flux in order to extract the energy barrier. ${ }^{13}$ The deposition process has been addressed by FIM, ${ }^{14}$ reflection high-energy electron dif- fraction (RHEED), ${ }^{15}$ and molecular dynamics (MD) simulations. ${ }^{16,17}$

Helium measurements can tell us something about the growth and the dynamics at low coverage. Several authors have successfully used the diffraction of a thermal helium beam to probe the sample surface during the diffusion of the adsorbates. ${ }^{18,19}$ The present paper shows that the measurement of the reflected specular helium intensity as a function of coverage and surface temperature can be used as a powerful method to study the surface dynamics during the early stages of growth. A first approach to this technique has been reported by Poelsema, Verheij, and Comsa, ${ }^{20}$ who have used the thermal energy atom scattering (TEAS) technique to measure the diffusion constants of $\mathrm{CO}$ on a $\mathrm{Pt}(111)$ surface. The latter is first prepared with a defined number of vacancies and $\mathrm{CO}$ molecules are adsorbed at a low surface temperature $(107 \mathrm{~K})$, where all mobility is frozen out. The specular helium intensity is recorded as a function of coverage, and after the deposition, as a function of temperature that is increased linearly. When the $\mathrm{CO}$ molecules become mobile they get pinned to the vacancies. The cross section for diffuse scattering of the vacancies overlaps with those of the molecules, which results in an increase of the reflected helium intensity due to the reduced coverage of the defectfree part of the surface. A model leads then to the determination of the activation barrier for the diffusion of the molecules. A similar method has been used by the same group to study the initial steps of growth of $\mathrm{Pt}$ on $\mathrm{Pt}(111) .^{21}$

The method proposed here uses a very similar argument, i.e., the change of the scattering cross section depending on the aggregation of the adparticles; however, in this case not only the initial slope but the whole shape of the deposition curve is analyzed carefully. Therefore the size distribution of the adparticles is simulated with a nucleation model. The principle of the measurement is simple. Incident atoms are condensed on the surface at a fixed temperature. During and after the deposition, the helium intensity specularly reflected from the surface of the sample is measured. The evolution of 
the helium intensity during and after the deposition can be compared with a simulation, which attributes a scattering cross section to each particle size. The particle size distribution is a function of time, of the integrated flux of silver atoms deposited, and of the temperature. It is assumed that the adatoms diffuse on the surface according to a law of activated random walk. The hopping frequency of the adatoms, taken as a free parameter, is then reported in an Arrhenius plot, which gives the diffusion barrier and the preexponential factor.

We report on measurements of the deposition process and the thermal diffusion of $\mathrm{Ag}$ on $\mathrm{Pd}(100)$ using the method described above. This open and isotropic substrate was chosen to be sure that the thermal mobility of adatoms is frozen in at liquid nitrogen temperature. In recent years, the adsorption of $\mathrm{Ag}$ on Pd surfaces has been studied by means of Auger electron spectroscopy, ${ }^{22-25}$ low-energy electron diffraction ${ }^{25}$ (LEED) and Auger electron diffraction, ${ }^{26}$ ultraviolet and $\mathrm{x}$-ray photoemission spectroscopy, ${ }^{27,25}$ work function measurements, ${ }^{28}$ thermal desorption, ${ }^{27}$ and thermal desorption of $\mathrm{CO}^{25}$ Most of the cited works deal with $\mathrm{Ag}$ coverage between $10 \%$ and several monolayers, adsorbed on the low index Pd surfaces at room temperature. On the (111) (Refs. 22, 23, 28 and 29) and (100) (Refs. 24 and 26) surfaces, the first $\mathrm{Ag}$ layer is observed to grow epitaxially at room temperature and up to $500 \mathrm{~K}$. On the less dense (110) surface, no intermixing is observed at room temperature, ${ }^{25,27}$ whereas annealing at $520 \mathrm{~K}$ produces interdiffusion of $\mathrm{Ag}$ and Pd atoms, and progressive enrichment of the Ag overlayer with $\mathrm{Pd}$, until at $800 \mathrm{~K}$ a surface alloy of composition $\mathrm{Ag}_{0.5} \mathrm{Pd}_{0.5}$ is reached.

$\operatorname{Pd}(100)$ has been the less studied surface. In Refs. 24 and 26 , evidence is given that a complete phase separation exists up to $500 \mathrm{~K}$. In addition to these experimental results, MD calculations ${ }^{30}$ yield a larger binding energy $(-2.67 \mathrm{eV})$ for $\mathrm{Ag}$ adsorption in the fourfold hollow surface site, than in the substitutional first layer site $(-2.08 \mathrm{eV})$, with a high barrier for the exchange process $(1.34 \mathrm{eV})$. The submonolayer growth regime at low temperature has not received much interest, and in particular for the (100) surface, to our knowledge, it has not been studied until now.

\section{MODEL}

The use of the specular reflected He intensity as a probe of a surface and its adsorbates relies on two facts. First, the high flatness of the electron density for metal surfaces of low Miller indexes causes their high reflectivity for $\mathrm{He}$ atoms of thermal energy. Second, the cross section of adsorbates for diffuse helium scattering is much larger than their "physical" size. The reflected helium beam is therefore sensitive to the presence and the distribution of very low coverage of adsorbates. Since we measure the very beginning of nucleation and growth, we stay in a regime where the clusters are very small. For these small structures we can consider that the only contribution to the reflected beam is the diffraction amplitude $A_{s}$ from the uncovered surface.

In the case of adsorbates randomly distributed on the surface and acting as perfectly diffuse scatterers, the relative specular He intensity is given by

$$
\frac{I}{I_{0}}=(1-\theta)^{\Sigma_{a}} .
$$

This is the so-called lattice-gas formula ${ }^{31}$ where $\Sigma_{a}=2 \sigma_{1}$ is defined as the macroscopic cross section of the adsorbate (related to the intensity), $\sigma_{1}$ the microscopic cross section, i.e., the surface perturbed by the presence of an adatom, $\theta$ is the coverage in monolayers (ML), and $\Sigma_{a}$ and $\sigma_{1}$ are measured in surface unit cells of $\operatorname{Pd}(100)\left(\sigma_{\operatorname{Pd}(100)}=7.56 \AA^{2}\right)$.

This description supposes explicitly a random distribution of the particles. As soon as there is a mobility of the adsorbed species together with an attractive interaction between them, this hypothesis is no longer fulfilled and we need another approach. ${ }^{32}$

A quite general description consists in making the list of all possible configurations of small structures on the surface, to multiply each configuration with its statistical occurrence and to calculate the corresponding cross section. In this case, the reflected helium intensity becomes

$$
\frac{I}{I_{0}}(\theta)=\left(1-\sum_{i}^{\%} \sigma_{i} n_{i}(\theta)\right)^{2} .
$$

where $n_{i}$ is the coverage of particles (number of particles per surface site) containing $i$ atoms and $\sigma_{i}$ the corresponding cross section (the problem of isomers with different cross sections will be discussed below).

In Eq. (2), only the reflection of the He beam coming from parts of the surface free of adsorbates is considered, whereas the contribution to the intensity from adparticles or islands is neglected. This is only possible for small coverages. This equation approaches the lattice-gas formula with $\Sigma_{a}=2 \sigma_{1}$ in the case of randomly distributed atoms with a large cross section.

In this paper we will use Eq. (2) to describe the He intensity measured in static and dynamic conditions, i.e., below and above the threshold of mobility of the adatoms. For this we have to estimate the cross sections $\sigma_{i}$ of adparticles containing various numbers of atoms, and compute the size distribution $n_{i}$ of the adparticles as a function of coverage and time.

\section{A. Cross section: The geometrical overlap approach}

In order to calculate the cross section of a particle we assume that the overlap approach is valid, ${ }^{31}$ i.e., that the cross section of a particle is numerically estimated by calculating the total area that is covered when the atomic cross section is associated to each of its atoms. We also assume (i) that the silver adatoms sit in epitaxial adlayer adsorption sites, this is supported by the fact that the growth of silver on $\operatorname{Pd}(100)$ begins with two epitaxial monolayers, as shown in Fig. 1; (ii) that the cross section can be approximated by a circular surface; (iii) that the clusters are two dimensional since the coverage is low (few percent of a monolayer). The value of the effective cross section per atom decreases rapidly as the number of atoms increases. This renders the specular $\mathrm{He}$ intensity very sensitive to the relative abundance of adatoms and small clusters on the surface; the present work takes advantage of this fact.

A complete description would take into account that different isomers exist for a given cluster size $i$. To simplify the 


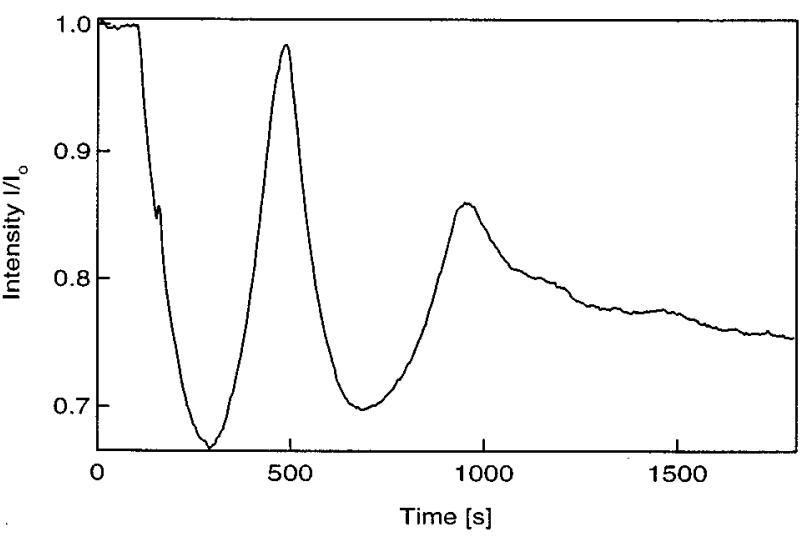

FIG. 1. Specular normalized helium intensity as a function of time for a deposition at $T_{s}=400 \mathrm{~K}$ and a Knudsen cell temperature $T_{\mathrm{Knu}}=1221 \mathrm{~K}$. We observe a Stranski-Krastanov type of growth, i.e., the completion of two epitactic layers followed by a threedimensional growth. The first maximum corresponds to the completion of the first monolayer, allowing a precise calibration of the incoming flux of $\mathrm{Ag}$ atoms.

description we have chosen for each size an isomer having a cross section that is close to the mean value of all compact isomer cross sections. For an atom attached to a step edge, the cross section has been chosen to be about $1 / 3$ of its value on a terrace because of the overlap with the cross section of the step.

\section{B. Size distribution: Rate equations}

To calculate the densities $n_{i}(\theta)$ of the $i$-mers, we use a rate equation approach known in the literature as thin-film nucleation theory. This theory has been developed and used by different authors ${ }^{33-36,6}$ (and included references). In the following, we describe the version of these equations used in this work; we discuss their validity and suggest some modifications. First we explain the static part of the equations, i.e., the description of the deposition process excluding any mobility due to the thermal energy, while in a second section we take into account the thermal mobility of the adsorbed atoms. Since we work at temperatures below and just above the mobility of the adatom, we neglect the two- and threedimensional evaporation of condensed atoms. In this respect, this approach is different from that given, for example, by Venables ${ }^{34}$ due to the particular nucleation conditions employed, i.e., low temperature and low coverage. Moreover we consider explicitly all different cluster sizes only up to a given size $i_{\max }$, since the cross section for diffuse scattering changes considerably for small clusters.

\section{Deposition process}

The deposition is characterized by a constant incoming flux of silver atoms $R(R=d \theta / d t=$ const $)$. The source term $s_{i}$ for a particular cluster containing $i$ atoms is given by the number of particles with $(i-1)$ atoms that collect an additional atom from the incoming flux. Obviously the source term of free adatoms corresponds to the ones that are not absorbed by larger particles. Hence (a)

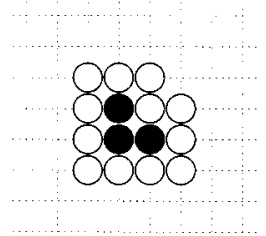

(c)

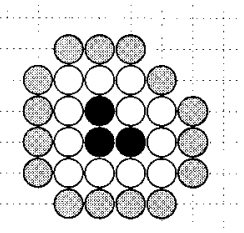

(b)

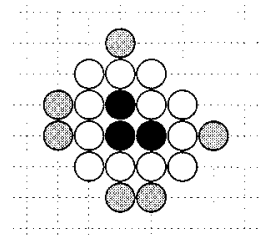

(d)

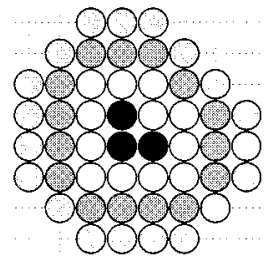

FIG. 2. View of a trimer with different capture zones. (a) Lattice gas (no mobility), (b) capture zone 1 short step (nearest neighbors), (c) capture zone 1 long step (nearest and next-nearest neighbors), (d) capture zone 2 steps.

$$
\begin{gathered}
s_{i}=\alpha_{i-1} n_{i-1} R, \quad i \geqslant 2, \\
s_{1}=R-\sum_{i \geqslant 2}^{\%} s_{i},
\end{gathered}
$$

where $\alpha_{i-1}$ is the number of sites, which corresponds to the capture zone of particles with $(i-1)$ atoms. Thus $s_{i}$ represents the capture rate of an impinging monomer by an existing $(i-1)$-mer.

We can now write the equation that describes the variation of the density of $i$-mers. One easily realizes that the pit term for the $i$-mer is also the source term of the $(i+1)$-mer, hence

$$
\frac{d n_{i}}{d t}=s_{i}-s_{i+1} .
$$

If the incoming atoms stay exactly on the site on which they are impinging the distribution of the atoms is random and Eq. (1) describes the dependence of the helium beam intensity on the coverage $\theta$. It has been shown ${ }^{14}$ that deposited atoms impinging on the proximity of a cluster are attracted toward it. We shall show later on that a single adatom can also attract an atom deposited in its neighborhood. ${ }^{37}$ This means that expression (1) is no longer valid whereas Eqs. (2) and (3) can be adapted to account for this phenomenon by simply adapting the size of the capture zones $\alpha_{i}$. Figure 2 shows a top view of a trimer surrounded by different sizes of the capture zones: all the atoms impinging on these capture zones move towards the already existing trimer. Figure 2(a) corresponds to the lattice-gas model (only the atoms impinging next to the existing particle are captured by it, there is no mobility). The other cases $b, c, d$ require a zero temperature mobility, which is not thermally activated, which we called neighbor driven mobility. ${ }^{37}$ Table I gives the values of $\alpha_{i}$ $(i=1-6)$ for the different sizes of capture zones.

\section{Diffusion}

If the temperature is high enough, the adparticles become mobile and this dynamical behavior modifies the particle size 
TABLE I. Capture zones $\alpha_{i}$ corresponding to the chosen shape of the $i$-mers. The columns (a) to (d) refer to the various types of capture zones shown in Fig. 2 in the case of the trimer.

\begin{tabular}{lclll}
\hline \hline & (a) & (b) & (c) & (d) \\
\hline$\alpha_{1}$ & 9 & 13 & 21 & 37 \\
$\alpha_{2}$ & 12 & 18 & 26 & 44 \\
$\alpha_{3}$ & 15 & 21 & 30 & 49 \\
$\alpha_{4}$ & 18 & 25 & 34 & 54 \\
$\alpha_{5}$ & 19 & 27 & 36 & 57 \\
$\alpha_{6}$ & 22 & 28 & 39 & 60 \\
\hline \hline
\end{tabular}

distribution. In the following we shall suppose that the particles follow a random walk with a hopping frequency $\nu_{i}$. On its way an adparticle can meet another one and coalesce in a larger particle. The creation and the disappearance of adparticles due to the collisions between them during their random walk can be described in terms of lifetimes: let $\tau_{i j}$ be the mean lifetime of a moving particle with $i$ atoms before it coalesces with a particle with $j$ atoms. This lifetime is defined by

$$
\tau_{i j}=\frac{\left\langle N_{j}\right\rangle}{\left\langle N_{v i}\right\rangle}
$$

where $\left\langle N_{j}\right\rangle$ is the average number of surface sites a particle has to visit in order to find a particle with $j$ atoms and $\left\langle N_{v i}\right\rangle$ the averaged number of surface sites visited per unit time by the particle with $i$ atoms. In a first approximation, $\tau_{i j}=1 /\left(\nu_{i} n_{j}\right)$, and the rate equation describing the life of a particle with $i$ atoms is

$$
\frac{d n_{i}}{d t}=s_{i}-s_{i+1}+\sum_{j} \sum_{k=i-j} \frac{n_{j}}{\tau_{j k}}-\sum_{j} \sum_{k}\left(\delta_{i j}+\delta_{i k}\right) \frac{n_{j}}{\tau_{j k}},
$$

where the third term describes the creation of an $i$-mer resulting from the diffusion and aggregation of atoms and smaller clusters, while the fourth term describes the destruction of an $i$-mer due to its merge with another particle. The terms expressing a finite lifetime of an adparticle related to its dissociation (for example, adatoms leaving a cluster) have been neglected in the present model.

Since the specular intensity is especially sensitive to the abundance of the smallest particles we restrict the system of rate equations (6) to the size of clusters with at most $i_{\max }=6$ atoms. In other words, we consider here the cases where the adatoms and the clusters containing up to six atoms are the dominant population on the surface. We call islands the larger clusters $(i>6)$, characterized by a mean size $l_{\text {island }}=\theta_{\text {island }} / n_{\text {island }}$ where $\theta_{\text {island }}$ and $n_{\text {island }}$ are, respectively, the coverage due to the islands and their number per unit area. The total coverage is thus $\theta=\sum_{i} i n_{i}+\theta_{\text {island }}$. The number of islands obeys the equation

$$
\frac{d n_{\text {island }}}{d t}=s_{i_{\max }+1}+\sum_{j}^{i_{\max }} \sum_{k>i_{\max }-j} \frac{n_{j}}{\tau_{j k}}
$$

and the coverage of atoms belonging to the islands is given by

$$
\frac{d \theta_{\text {island }}}{d t}=s_{i_{\max }+1}\left(i_{\max }+1\right)+\sum_{j}^{i_{\max }} \sum_{k>i_{\max }-j} \frac{n_{j}}{\tau_{j k}}(j+k) .
$$

So far the surface has been supposed to be perfect. In reality this ideal case is never met and it is well known that defects can highly affect the crystal growth. In our case we must consider, for instance, that diffusing adparticles can move to surface steps where they condense. The step density $n_{\text {step }}$ is a given characteristic of the sample that we cannot measure in our experiment. We can only estimate that this value is similar to that obtained by STM measurements taken on a crystal surface prepared according to an identical procedure from the same single crystal. ${ }^{38}$ In that case the step density results to be $n_{\text {step }}=1 / 500$; i.e., every atom over 500 is a step atom. It is supposed constant during the experiments. The condensation of the adparticles along the steps is a onedimensional problem. An adparticle containing $i$ atoms has a limited lifetime $\tau_{i \text { step }}$ due to trapping by the steps, which is defined by the averaged number of surface sites between two steps divided by the averaged number of surface sites visited per unit time by the particle (in a first approximation $\left.\tau_{i \text { step }}=2 / \nu_{i} n_{\text {step }}\right)$. We suppose here that the steps are randomly distributed, which is realistic if we have a signal averaged over macroscopic distances. The number of atoms of all particles condensing on the steps is then given by $d n_{\text {step }} / d t=\Sigma_{i}\left(n_{i} / \tau_{i \text { step }}\right) i$.

Taking the condensation on steps into account, the rate equation (6) for particles with $i$ atoms becomes finally

$$
\begin{aligned}
\frac{d n_{i}}{d t}= & s_{i}-s_{i+1}+\sum_{j} \sum_{k=i-j} \frac{n_{i}}{\tau_{i j}} \\
& -\sum_{j} \sum_{k}\left(\delta_{i j}+\delta_{i k}\right) \frac{n_{j}}{\tau_{j k}}-\frac{n_{i}}{\tau_{i \text { step }}} .
\end{aligned}
$$

Two corrections have to be introduced:

(a) Capture zones. In the above description, each particle occupies only one site, which is not the case in reality. The area associated with a particle of size $i$ is the dimension of its capture zone $\alpha_{i}$. An easy way to account for this problem is to come back to the definition of the lifetime $\tau_{i j}$, which depends on the mean number of sites $\left\langle N_{j}\right\rangle$ a particle has to visit to find a particle $j$. It was estimated to $1 / n_{j}$ but this value can be corrected to ${ }^{39}$

$$
\left\langle N_{j}\right\rangle=\frac{1}{n_{j}}-\alpha_{j} .
$$

This correction is mainly important for a high density of particles or equivalently at low dynamics.

(b) Memory of the system. Let us consider, for example, the collision between a monomer and a dimer and suppose that the mobility is higher for the monomer than for the dimer. This means that the monomer will move in a nearly fixed environment of dimers; therefore it will visit a new site after each hop in the very beginning of its existence, but later it will have a high probability to fall on a site already visited in the past that is clearly free of dimer. The dimer moving towards a monomer will not experience the same phenomenon since its environment of monomers cannot be considered as fixed. 


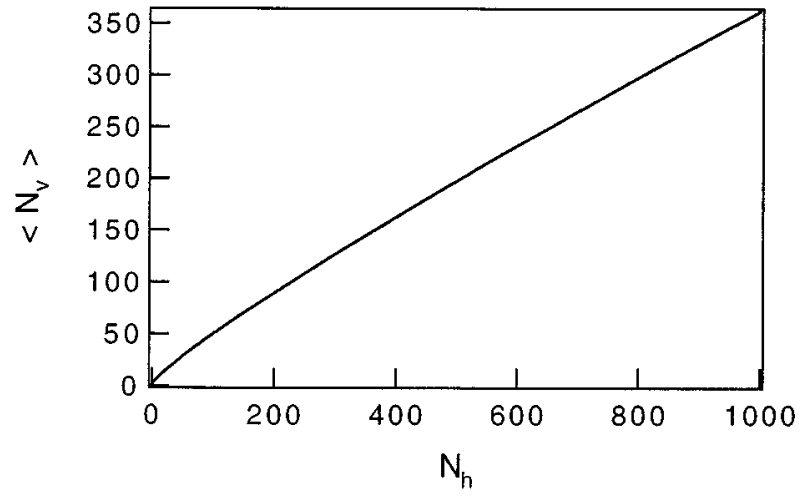

FIG. 3. Mean number of visited sites $\left\langle N_{v}\right\rangle$ vs number of hops $N_{h}$ for a particle performing a random walk in two dimensions.

Again we are coming back to the definition of the lifetime $\tau_{i j}$ given by Eq. (5). Under the hypotheses that only particles $i$ are mobile and there is no source term for particles $j$, this problem can be considered as a random walk. Such a system has been studied in the literature ${ }^{40,41}$ and in two dimensions there is no analytical solution for the mean number of visited sites $\left\langle N_{v}\right\rangle$ after $N_{h}$ hops, only the asymptotic value is proposed. ${ }^{41}$ An easy way to calculate $\left\langle N_{v}\right\rangle$ is to use a Monte Carlo simulation; Fig. 3 shows the result of an average over $10^{6}$ runs. We can define the function

$$
f_{v}=\frac{\left\langle N_{v}\right\rangle-1}{N_{h}}
$$

so that the number of newly visited sites after $N_{h}$ hops equals $N_{h} f_{v}$.

For a particle $i$ created at $t^{\prime}=0$, its lifetime before meeting a particle $j$ becomes [Eqs. (10) and (11)]

$$
\tau_{i j}=\left(\frac{1}{n_{j}}-\alpha_{j}\right) \frac{1}{\nu_{i} f_{v}\left(\nu_{i} t^{\prime}\right)} .
$$

We have now a precise description of the lifetime of a particle. However, this description is not easy to use since the lifetime depends explicitly on the time $t^{\prime}$ and all particles $i$ are not created at the same time. In order to account for this correction we would have to convolute our equations with this lifetime, which would complicate their integration. Moreover the hypotheses on which the description is built are often not satisfied (particles $j$ immobile, no source term especially during the deposition). For these reasons we chose to switch on the memory of the system only at the end of the deposition and only for very mobile particles colliding with less mobile particles. We reach here the limit of our description in rate equations. For example, the depletion of atoms in the proximity of steps or big islands is neglected, causing an overestimate of the capture rate by the steps at low island density. ${ }^{42}$ A Monte Carlo study would not have the same limits but the computing effort it requires is more important.

\section{Simulations}

The system of Eq. (10) for each size between one and six atoms together with the equations describing the growth of islands [Eq. (7) and the condensation on steps] have been solved numerically. The value of the time increment in the numerical calculation must be chosen by considering the hopping frequency $\nu_{i}$ and also the silver flux $R$. For example, a good stability is obtained for $R=10^{-3} \mathrm{ML} / \mathrm{s}$, and $\nu_{1}=1 \mathrm{~s}^{-1} \quad\left(\nu_{i}=0\right.$ for $\left.i>1\right)$ with a time increment $\Delta t=0.05 \mathrm{~s}$. We performed simulations for hopping frequencies varying from $\nu_{1}=10^{-3} \mathrm{~s}^{-1}$ up to $\nu_{1}=100 \mathrm{~s}^{-1}$; in the high dynamic range we reach the limit of the validity of the simulations due to the increasing importance of the condensation of the particles on the steps. Figure 4 shows an example of a particle size distribution as a function of coverage for three different atomic hopping frequencies: (a) $\nu_{1}=0.1$ $\mathrm{s}^{-1}$; (b) $\nu_{1}=1 \mathrm{~s}^{-1}$; (c) $\nu_{1}=10 \mathrm{~s}^{-1}$. The condensation on the islands and on the steps is also displayed. Figure 4(d) gives the resulting helium intensity $I / I_{0}(\theta)$ expected in an experiment, and also the lattice gas behavior.

Stowell $^{43}$ showed that there is a linear relation between the logarithm of the island density and the diffusion energy of the monomer $\ln n_{\text {island }} \sim\left(E_{d_{1}} / T+\right.$ const $)$. It is worth noting that this relation is nicely reproduced by our model. Several characteristics appear in the curves of Fig. 4.

(i) The lifetime is coverage dependent. However, one observes a similar behavior at very low coverage (up to about $0.7 \%$ ) for the three hopping frequencies considered. They correspond to a domain of low dynamics. The same value of the atomic cross section can be deduced from the initial slope of the $I / I_{0}(\theta)$ curve [Fig. 4(d)]. As the coverage increases, the lifetime of the adatom is strongly reduced, and larger aggregates and islands are formed. This leads to a deviation from the lattice-gas behavior of the relative helium intensity $I / I_{0}$ as a function of coverage. This effect depends strongly on the hopping frequency, hence on the temperature. For example, a deviation of $2 \%$ from the lattice-gas intensity curve is obtained at a coverage $\theta=0.06$ if $\nu_{1}=0.1$ $\mathrm{s}^{-1}, \theta=0.02$ if $\nu_{1}=1 \mathrm{~s}^{-1}$, and $\theta=0.009$ if $\nu_{1}=10 \mathrm{~s}^{-1}$.

(ii) The role of the steps in the dynamics depends also strongly on the temperature. When $\nu_{1}=0.1 \mathrm{~s}^{-1}$, the proportion of adatoms that condense on steps is almost negligible. However, when $\nu_{1}>1 \mathrm{~s}^{-1}$ a significant part of the deposited atoms is on the steps and this part increases with coverage. At large $\nu_{1}$ this behavior constitutes one of the limits of the model, since we do not know how the growth proceeds along the steps and no coherence effects in the scattering of the helium atoms have been taken into account in the calculation of the intensity reflected by the surface of the sample.

The above model has also been applied to simulate the specular He intensity after the Ag deposition has been stopped.

\section{EXPERIMENT}

The measurements are carried in a UHV-molecular-beam apparatus, which has been described in detail elsewhere. ${ }^{44}$ It consists of an analysis chamber with a base pressure in the low $10^{-11}$ mbar range coupled to a differentially pumped He nozzle beam and a detector located in a separately pumped chamber. The $\operatorname{Pd}(100)$ crystal is mounted on a sample holder allowing for $x y z$, polar angle, and tilt displacement. The sample temperature is measured between 80 and $1300 \mathrm{~K}$ with a thermocouple spot welded on the crystal, 


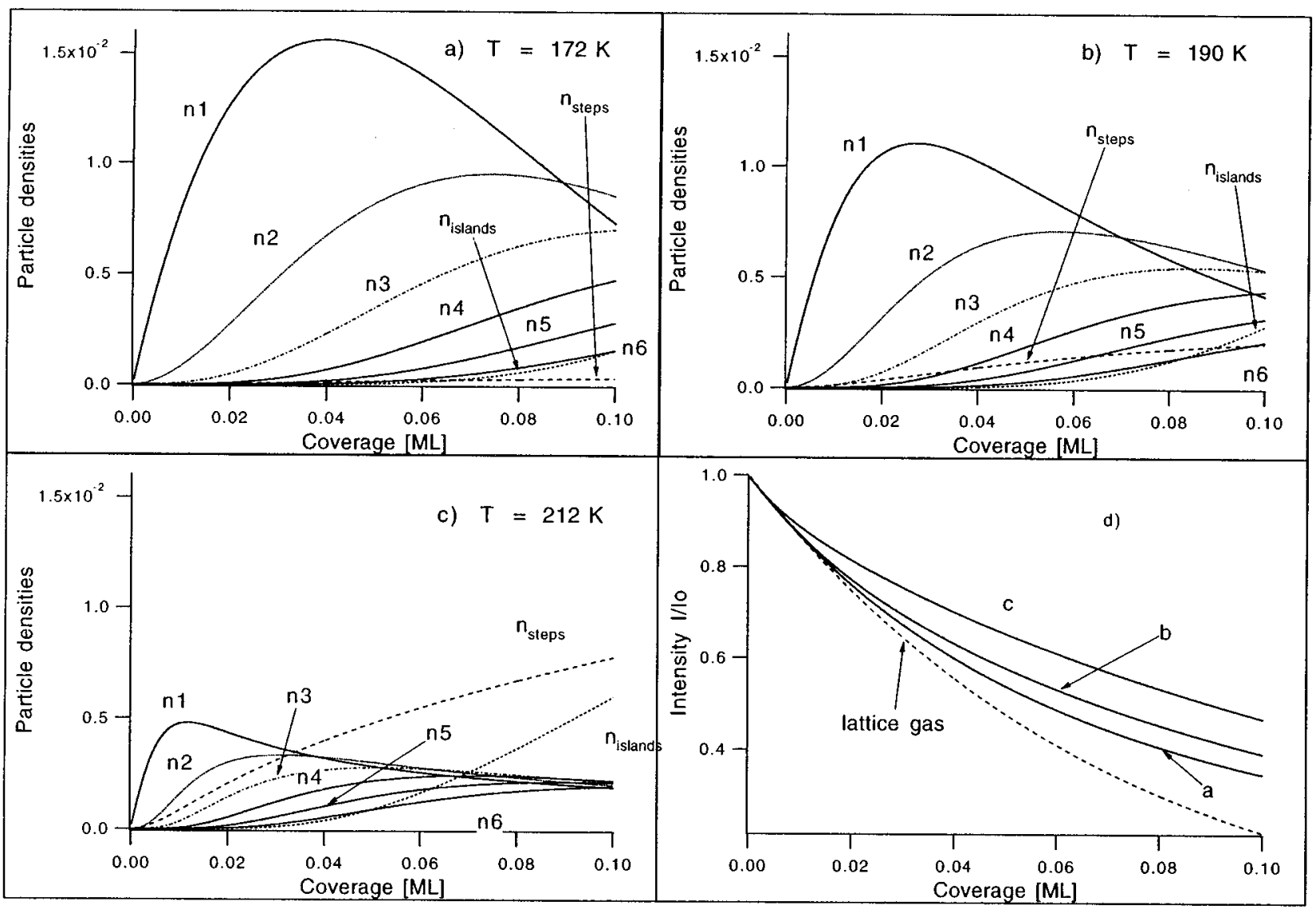

FIG. 4. Calculated atom and cluster densities as well as corresponding helium signal (d) for the different dynamical parameters: (a) $\nu_{1}=0.1 \mathrm{~s}^{-1}$, (b) $\nu_{1}=1 \mathrm{~s}^{-1}$, and (c) $\nu_{1}=10 \mathrm{~s}^{-1}$ ( $\nu_{i}=0$ for $i \geqslant 2$ in all cases). The corresponding substrate temperatures are shown at the top right corner of the figures.

and it can be varied by electron beam heating and liquid nitrogen cooling. Repeated sputtering $\left(\mathrm{Kr}^{+}, 1000 \mathrm{eV}, 1\right.$ $\mu \mathrm{A} \mathrm{cm}^{-2}$ ) and oxydation cycles have initially cleaned the crystal surface. Everyday cleaning of the surface is also performed by short $(15 \mathrm{~min})$ sputtering periods at room temperature, followed by an annealing of $3 \mathrm{~min}$ at $1000 \mathrm{~K}$ and $15 \mathrm{~s}$ at $1300 \mathrm{~K}$. A temperature stabilized Knudsen cell directed almost normal to the surface has been used to deposit silver fluxes ranging from $10^{-4}$ to $10^{-2} \mathrm{ML} / \mathrm{s}$. The geometry allows for the measurement, during the deposition, of the intensity of a helium beam specularly reflected by the sample surface. The supersonic He beam is emitted by a nozzle at 77 $\mathrm{K}$, then it crosses two stages of collimation (differential pumping) and it hits the surface on a spot of $0.7-\mathrm{mm}$ diameter with an incidence of $64^{\circ}$ and an intensity of $1.6 \times 10^{15}$ atom $\mathrm{s}^{-1}$. The transfer width for this system is estimated to $50 \AA$ A. A quadrupole mass spectrometer located in another separately pumped chamber detects the specular reflected $\mathrm{He}$ beam. The precise calibration of the silver flux is based on depositions between 300 and $500 \mathrm{~K}$, where the time dependence of the relative specular He intensity $I / I_{0}$ presents two successive maxima before decreasing monotonically, as shown in Fig. 1 ( $I_{0}$ is the specular He beam intensity of the clean surface). They correspond to the growth of the first two epitaxial monolayers, in agreement with other authors. ${ }^{26} \mathrm{We}$ have taken the time needed to complete the first monolayer to calibrate the Knudsen cell flux as a function of temperature, and consequently the silver coverage $\theta$ on the $\operatorname{Pd}(100)$ surface. The He intensity of the first maximum equals approximately the intensity corresponding to the bare surface indicating that the first silver layer is well completed before the beginning of the growth of the second layer. Notice that a similar behavior does not seem to happen with the growth of the second silver layer, where the time needed to reach the second maximum exceeds somewhat the time of the first layer growth and the intensity of the second maximum is reduced. We probably observe there the beginning of the three-dimensional growth characteristic of the StranskiKrastanov growth mode. This is in agreement with previous studies of this system. ${ }^{24}$

At low temperature of the substrate we measure a small contamination of the sample due to the residual gas in the chamber. We have checked that this contamination does not come from the He beam. It produces a $0.5 \%$ signal reduction per minute, which translates into a pollution of the substrate of $5 \times 10^{-4} \mathrm{ML} / \mathrm{min}$; this corresponds to a background pressure of $5 \times 10^{-12}$ mbar if we assume a sticking coefficient of 1. For each measure the signal is recorded for about $150 \mathrm{~s}$ before starting the deposition to have a measure of the contamination. The measurements are then corrected to take into account this pollution by describing it through a decreasing exponential. 


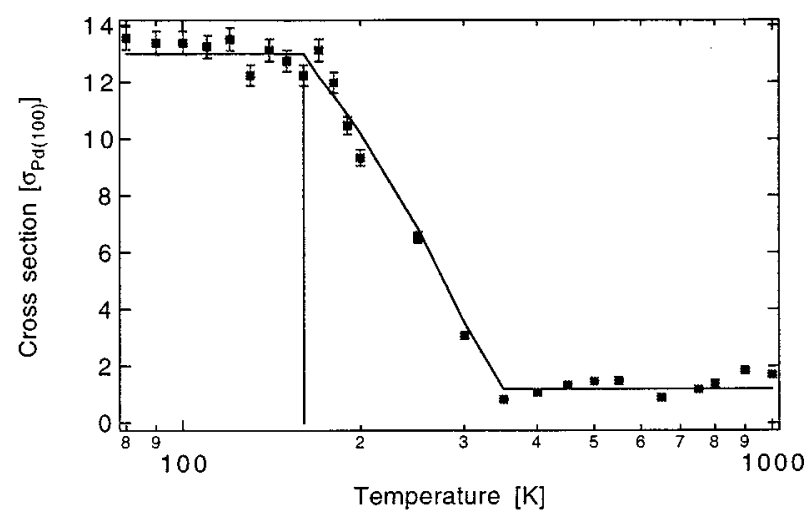

FIG. 5. Cross section $\Sigma_{l g}$ fitted on the intensity vs coverage curves between 0 and $1 \% \mathrm{ML}$ as a function of temperature. The deposition rate is $R=1 / 1043 \mathrm{ML} / \mathrm{s}$.

\section{RESULTS AND DISCUSSION}

Figure 5 shows the lattice-gas cross section $\Sigma_{\text {lg }}$, i.e., the cross section measured by fitting the measured intensity with the lattice-gas formula (1) between 0 and $1 \%$ ML coverage. This gives an effective cross section that varies with the deposition temperature $T_{s}$ comprised between 80 and 1000 $\mathrm{K}$.

The cross section is constant below $160 \mathrm{~K}$, which shows that in the time scale of the experiment (typically $10 \mathrm{~s}$ ) the morphology of the surface does not change with temperature. This means that the mobility of atoms and small particles is negligible in this temperature range. Above $T_{s}=160 \mathrm{~K}$, we observe a rapid decrease of $\Sigma_{\mathrm{lg}}$, coming from the increasing mobility of the $i$-mers with temperature. Atoms group together due to their high mobility and form bigger structures; there are fewer point defects and a lower attenuation of the reflected signal. In Sec. IV A we consider the measurements at low temperature while the diffusion measurements at higher temperature are discussed in Sec. IV B.

\section{A. Static measurements}

Figure 6 shows a typical deposition curve obtained at low deposition temperature $\left(T_{s} \leqslant 160 \mathrm{~K}\right)$. It is evident that it cannot be fitted with the lattice-gas model, which implies that the distribution of atoms cannot be considered uniform. In other words the aggregates are not formed statistically by incident atoms, but a mobility after impact has to be considered.

Two different mechanisms have been proposed in the literature to explain the mobility of adatoms on a surface at temperatures low enough to prevent the thermal diffusion of the atoms, the so-called transient mobility model and what we introduced as the neighbor driven mobility model. ${ }^{37}$

The transient mobility model relies on the fact that the energy of an incident atom before its thermalization is high in comparison with typical diffusion barriers on a metallic surface so that the adsorption energy is partially converted into kinetic energy parallel to the surface. To check if our measurements can be reproduced by taking in account such a phenomenon, we wrote a Monte Carlo code where each atom deposited on the surface is permitted to make a given num-

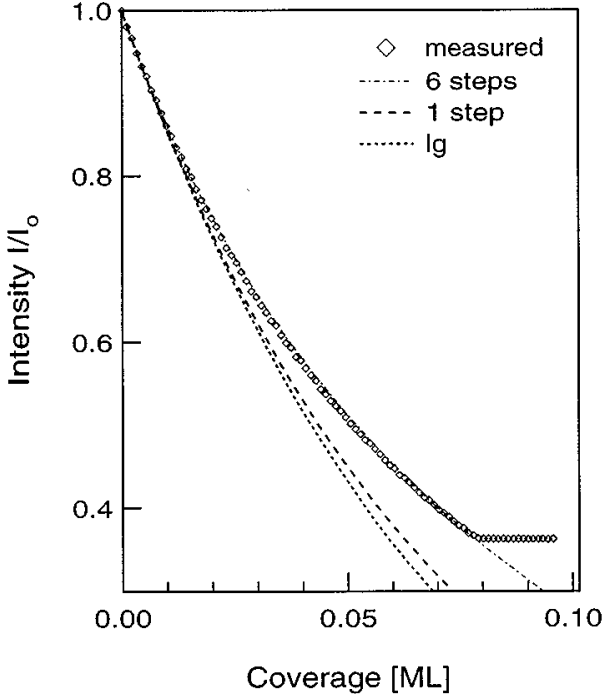

FIG. 6. Helium specularly reflected intensity for a deposition at $T_{s}=80 \mathrm{~K}$, compared to a Monte Carlo simulation of transient mobilities of one and six steps in random directions.

ber of hops in random directions. The result of this simulation is shown in Fig. 6 for one and six hops. Our analysis indicates that each atom has to make up to six hops in order to obtain a perfect agreement between the calculated and measured signal.

The neighbor driven mobility model takes into account the fact that the presence of an adatom or cluster on the surface modifies the potential around it and thus the barrier for the capture of an incoming adatom. In this description all incoming atoms deposited at a given distance of an already existing particle move toward it. This defines the capture zones that have been introduced previously (see Sec. II B 1 and Fig. 2).

In order to decide which of the different capture zones better describes the measure, we calculate the size distribution of the particles using Eqs. (3) and (4). Then we fit the atomic cross section on the experimental points for a coverage between 0 and $7 \%$ ML. Figure 7 shows the relative difference between the fit and the measured intensity for each model. This analysis shows clearly that only the model with one step (nearest-neighbor and next-nearest-neighbor sites) is able to reproduce correctly the experimental measurements. The atomic cross section extracted from this fit is equal to $\Sigma_{1 l}=2 \sigma_{1}=14.4 \pm 0.7 \sigma_{\mathrm{Pd}(100)}=109 \pm 5 \AA^{2}$. The same result is observed for all depositions between $T_{s}=80$ and $160 \mathrm{~K}$, which means that the modification of the potential responsible for such a mobility is important. The barrier has to be at least twice lower than on a nude surface in order to explain the occurrence of a mobility already at $T_{s}=80 \mathrm{~K}$.

\section{Discussion}

This analysis indicates that our measurements are well reproduced either with a model allowing for six steps of transient mobility or by a model that considers the mobility of neighbor atoms of one site (long and short steps).

Some experimental results have been explained with a transient mobility. Abrams and Weiss ${ }^{45}$ have observed a transient mobility for Xe on $\mathrm{Pt}(111)$ with $\mathrm{STM}$ at $T_{s}=4 \mathrm{~K}$, a 


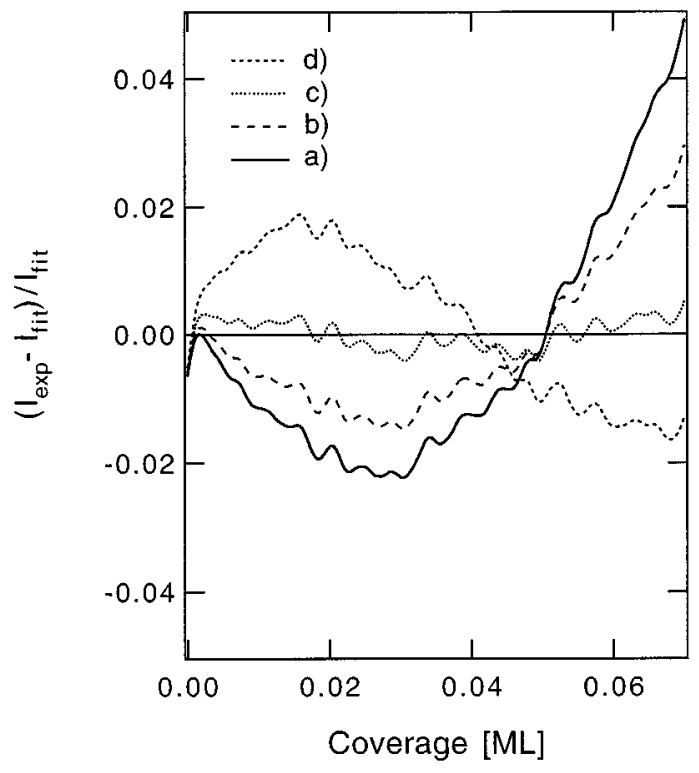

FIG. 7. Relative difference ( 0 to $7 \%$ ML) between the best fits on the experimental data with different capture zones (see text). Experimental data refer to $T_{s}=80 \mathrm{~K}$ and $R=1 / 1043 \mathrm{ML} / \mathrm{s}$.

temperature at which the diffusion is frozen. Egelhoff and $\mathrm{Jacob}^{15}$ proposed the same idea to explain oscillations of the RHEED signal at $77 \mathrm{~K}$ for the growth of $\mathrm{Fe}$ and $\mathrm{Cu}$ on $\operatorname{Ag}(100)$; however, this explanation has been criticized and completed after further investigations by other authors. ${ }^{46,47}$

Experimental results as well as several numerical simulations tend, however, to show that there is no transient mobility for deposition of metals on metals. Wang and Ehrlich ${ }^{48}$ showed with FIM measurements that atoms adsorb with an equal proportion on fcc and hep sites of a (111) surface of Ir, which do not have the same binding energy. They conclude that atoms adsorb on the nearest site of the impact point; if there was a transient mobility atoms would preferably go on the energetically more favorable sites. Sanders and DePristo $^{16}$ by studying the homoepitaxy on different (100) surfaces of transition metals have come to the conclusion that there is no transient mobility except for silver where the effect is small. Blandin and Massobrio ${ }^{49}$ find that $\mathrm{Ag}$ atoms deposited with an incident energy of $5 \mathrm{eV}$ move a distance of at most $5 \AA$ on Pt(111). De Lorenzi and Ehrlich ${ }^{17}$ studied the energy transfer for the adsorption of an atom on a crystal by using Lennard-Jones potentials. They show that the energy transfer is again very effective, only the atoms that experience a head-on collision with a surface atom can show a very small mobility. Stoltze and Norskov ${ }^{47}$ showed that in the case of the homoepitaxy of $\mathrm{Cu}(111)$, the dissipation of energy is sufficiently quick that $\mathrm{Cu}$ atoms accommodate on clusters constituted of three atoms. These results and the high number of hops required to explain our results by a transient mobility lead us to conclude that this explanation does not hold.

The neighbor driven mobility model, which assumes a modification of the potential energy surface around an adatom seems more realistic. However, as we have already pointed out, the modification of the potential has to be important to account for the measurements at $T_{s}=80 \mathrm{~K}$. Diffusion and deposition experiments reported by Wang and

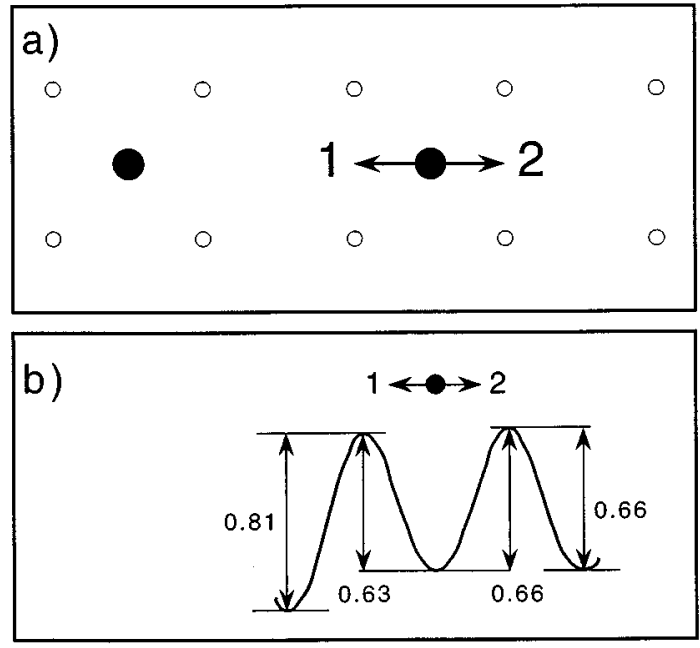

FIG. 8. Potential energy profile for an adatom in the proximity of another adatom on a $\operatorname{Pd}(100)$ surface. The indicated values are in $\mathrm{eV}$.

Ehrlich $^{50,14}$ confirm this idea. They show that the barrier in the proximity of a cluster has to be 4 times lower than the usual one to account for their low-temperature deposition measurements.

In order to test this idea in the case of our system, we used a molecular dynamic computer code using the embedded atom method (EAM) scheme ${ }^{51}$ to calculate static diffusion barriers in the proximity of atoms or clusters on the surface.

The substrate is composed of ten atomic layers [(100) orientation of a fcc crystal] of 50 atoms each. Periodic boundaries are set in the $x$ and $y$ directions. Diffusion barriers are computed by depositing on the substrate one or several atoms and letting the system relax at $0 \mathrm{~K}$. One of the atoms is then displaced along a given path. Its $x$ and $y$ coordinates are fixed while its $z$ position is allowed to relax. The total energy of the system gives then the energy barrier along the chosen path.

We find a calculated static diffusion barrier for a free $\mathrm{Ag}$ adatom on the surface of $\operatorname{Pd}(100)$ equal to $E_{d 1}=0.66 \mathrm{eV} ;{ }^{52}$ the calculated potential profile for an adatom approaching another atom adsorbed on the surface is shown in Fig. 8. The calculation gives a lowering of the diffusion barrier by about $5 \%$. Diffusion barriers for adatoms approaching small islands have also been studied extensively and show basically that the reduction of the barrier is at most of $11 \%$, which is clearly insufficient to explain our data. These calculations show also that the barrier to move an atom with a long bond (next-nearest-neighbor position) to form a short bond (nearest-neighbor position) is about 35\% lower than the simple diffusion barrier. This justifies our choice of compact structures for the clusters in our model.

It seems to be a general trend of the EAM calculation to show no substantial change of the diffusion barrier near a step. Liu and Adams, ${ }^{53}$ for example, calculated static diffusion barriers for diffusion of a $\mathrm{Ni}$ adatom near a step on (111), (110), and (100) surfaces of $\mathrm{Ni}$ by using an EAM scheme. In the case of the (100) surface the barrier is lowered by about $5 \%$ compared to the free adatom. For the (111) surface, the decrease is $30 \%$ to $60 \%$ for atoms in the third- 


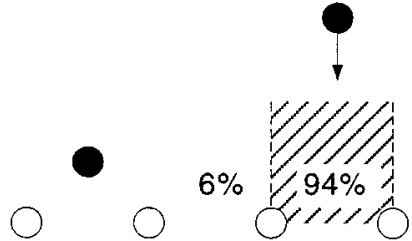

FIG. 9. Simulation of Ag atoms deposited in the proximity of a preexisting Ag atom (in black) on the surface (surface Pd atoms in white). The impact points are chosen randomly in the marked area. Most of them stay in the site where they landed, however, some of them $(\sim 6 \%)$ move towards the preadsorbed Ag atom. Movements towards any other site have not been observed.

neighbor position while atoms in the second-neighbor position are not stable. This could qualitatively explain the results of Wang and Ehrlich; ${ }^{14}$ the effect is, however, too low to account quantitatively for their results since the observed attraction implies still lower energy barriers.

To test for an eventual combined effect of the transient mobility and a deformation of the potential surface, we simulate depositions of $\mathrm{Ag}$ atoms on $\mathrm{Pd}(100)$ in the presence of a preadsorbed $\mathrm{Ag}$ atom. The silver atoms are impinging with normal incidence on a surface at $0 \mathrm{~K}$ (and at $80 \mathrm{~K}$, which turns out not to change the outcome of the simulations) and an initial energy of $0.16 \mathrm{eV}$. The initial positions have been chosen randomly in the unit cell represented on Fig. 9. In order to obtain a reliable statistics, we performed 50 simulated depositions. The atoms are accelerated towards the surface and gain 2.98-eV adsorption energy; they collide with the surface and dissipate their energy and the simulation is stopped once they are thermalized. Figure 9 shows the repartition in the positions of the atoms at the end of the runs. Some atoms have moved towards the already adsorbed atom on a neighbor site. This is the only mobility that is observed; i.e., the incident atoms never move on other neighbor sites. There is an effect, however, it is not in quantitative agreement with the experiment: the model we used to explain our experimental data requires that all the incident atoms move towards the adsorbed atom, while the simulation shows that only $6 \%$ of the atoms move out of the unit cell in which they impact, and these are the atoms which land close to the border of the unit cell.

We have then to conclude that the EAM calculations are not quantitatively consistent with an explanation of our experimental results or those reported by Wang and Ehrlich ${ }^{14}$ by a deformation of the potential surface. This could be explained by the inaccuracy of the EAM potentials at low coordination. ${ }^{54}$ Moreover the EAM scheme tends to overestimate the efficiency of energy dissipation. ${ }^{55}$ Slightly longer relaxation times could sensitively increase the small amount of moving atoms found in this calculation.

\section{Conclusions}

We have experimental evidence that there is a mobility of atoms at much lower temperature than the activation temperature for thermal diffusion and the measurements can be reproduced by considering that all silver atoms deposited in a zone of about $6.1 \AA$ around a preexisting atom on the sur-

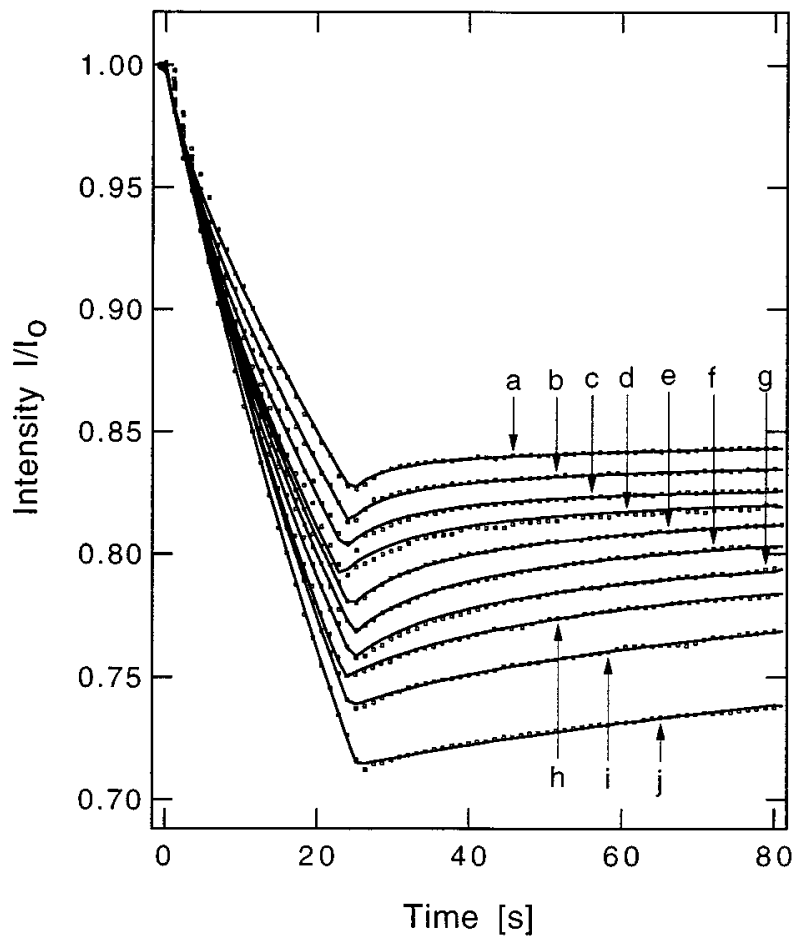

FIG. 10. Comparison between the experimental values and the model calculation (with corrections: capture zones and memory of the system) fitted on these experimental points. The fit parameters are $\nu_{1}$ and $\nu_{2}$. The measurements have been carried out at different surface temperatures $T_{s}$ : (a) $225 \mathrm{~K}$, (b) $220 \mathrm{~K}$, (c) $215 \mathrm{~K}$, (d) 210 $\mathrm{K}$, (e) $205 \mathrm{~K}$, (f) $200 \mathrm{~K}$, (g) $195 \mathrm{~K}$, (h) $190 \mathrm{~K}$, (i) $185 \mathrm{~K}$, and (j) 180 $\mathrm{K}$.

face are captured by the adatom. This mobility is similar to the one reported by Wang and Ehrlich ${ }^{14}$ but this time a single adatom seems to be sufficient to modify the potential energy surface seen by a second adatom.

Molecular dynamic calculations give qualitative insight into the processes involved. The lowering of the diffusion barrier due to the presence of an adsorbate combined with a transient mobility seems to be the most probable explanation for these experimental findings. Either of these two mechanisms taken individually would require unrealistic assumptions on the lowering of the potential barrier or on the thermalization time of the impinging adatoms.

\section{B. Dynamical measurements}

At a substrate temperature higher than $160 \mathrm{~K}$, the observed He intensity deviates more and more from the latticegas behavior; the slope of the curve, and hence the apparent cross section $\Sigma_{a}$, decrease with increasing temperature (see Fig. 5).

Figure 10 shows typical depositions. The signal decreases with increasing coverage; at a coverage of $\theta=2.3 \% \mathrm{ML}$ the deposition is stopped and the signal raises again due to the mobility of the atoms forming larger structures. The intensity as a function of time can be simulated with the kinetic model developed above by computing the size distribution of particles [see Eqs. (2) and (7) and the condensation on steps]. If we assume that particles larger than the dimer are immobile 


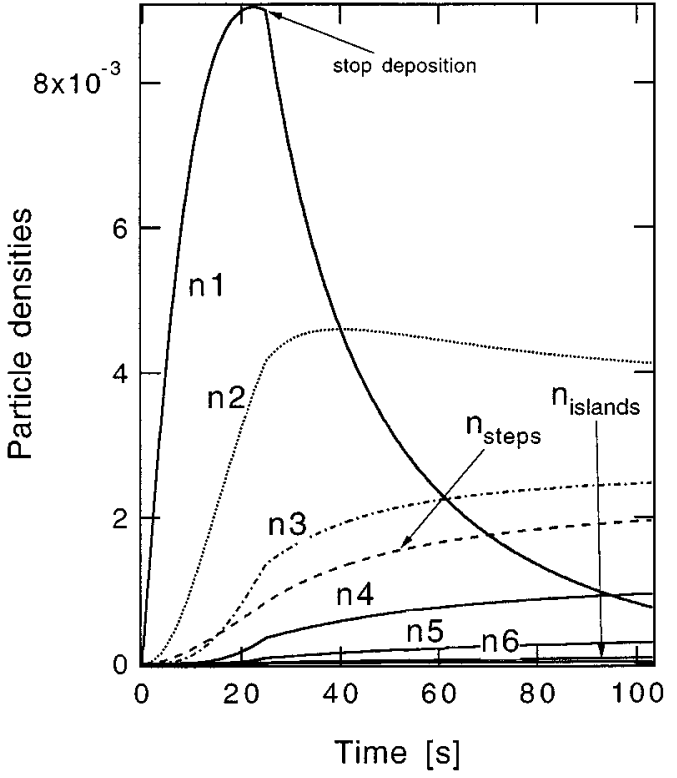

FIG. 11. Calculated particle size distribution for a typical deposition: the silver flux is switched on at $t=0$ and turned off at a coverage of $\sim 2.3 \%$ ML. A fit with the hopping frequencies of the monomer and dimer is performed and the resulting calculated intensity is shown in Fig. 10(f). Particle density is in units of surface density of $\operatorname{Pd}(100)$ sites $\left(1.3 \times 10^{15} \mathrm{~cm}^{-2}\right), \quad T_{s}=200 \mathrm{~K}$, $R=1 / 1043 \mathrm{ML} / \mathrm{s}$.

$\left(\nu_{i}=0\right.$ for $i \geqslant 3$ ) and that the atomic cross section $\Sigma_{a}$ has the value determined above, we can fit the data points with $\nu_{1}$ and $\nu_{2}$ as the only free parameters; the resulting computed intensity is represented in continous lines in Fig. 10. The agreement with the data points is excellent. Figure 11 shows the densities of adparticles, the number of atoms condensed on islands and on steps as a function of time for a deposition at $T_{s}=200 \mathrm{~K}$, and a flux $R=1 / 1043 \mathrm{ML} / \mathrm{s}$. These values result from a fit that gives a hopping frequency of the adatoms $\nu_{1}=3.8 \mathrm{~s}^{-1}$ for this particular case; the hopping frequency of the dimers $\nu_{2}$ is found to be close to zero.

This procedure can be repeated for different temperatures; the results of the fits are given in Fig. 10. We can then plot the logarithm of the frequency, $\ln \nu_{1}$, versus $1 / T_{s}$ and obtain the Arrhenius representation in Fig. 12. We can see that the data points are on a straight line in agreement with a model of first-order thermal activation:

$$
\nu_{1}=\nu_{01} \exp \left(\frac{-E_{d_{1}}}{k T}\right) \text {. }
$$

We obtain from the fit

$$
\nu_{0}=8 \times 10^{9} \mathrm{~s}^{-1}, \quad E_{d 1}=0.37 \pm 0.03 \mathrm{eV} .
$$

This result is obtained by using the corrections (capture zones and memory of the system) discussed above; however, it depends only very slightly on these corrections. ${ }^{56}$

\section{Discussion}

We restricted the analysis of our results to a range of temperatures where the mobility remains low, in order to maintain a regime of nucleation of small clusters and where

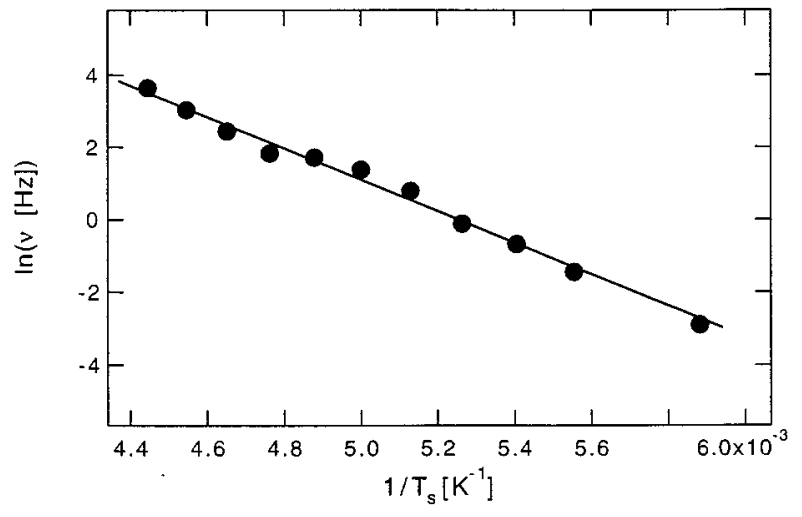

FIG. 12. Arrhenius plot resulting from the fitted parameter $\nu_{1}$. The fitting of a straight line (continuous line) gives the following diffusion parameters $\nu_{0}=8 \times 10^{9} \mathrm{~s}^{-1}$ and $E_{d}=0.37 \mathrm{eV}$.

the condensation on steps is small. This presents the double advantage of giving a high variation of the reflected helium beam and to be univoque (i.e., that we do not have to consider the difference between the growth of dendritic structures on the steps and big smooth islands); this is essential since our investigation tool does not give a local view of the surface, such as, for example, STM.

There is to our knowledge no other experimental determination of the diffusion parameters of $\mathrm{Ag}$ on $\mathrm{Pd}(100)$. The system $\mathrm{Cu} / \mathrm{Pd}(100)$ has been studied by $\mathrm{Hahn}^{38}$ with STM and a diffusion barrier of $0.28 \mathrm{eV}$ has been measured. LEED measurements on the homonuclear system $\mathrm{Pd} / \mathrm{Pd}(100)$ realized by Evans, Flynn-Sanders, and Thie ${ }^{57}$ show a barrier of $0.56 \mathrm{eV}$ if a preexponential factor of $5 \times 10^{12} \mathrm{~s}^{-1}$ is supposed. Venables and co-workers ${ }^{58,59}$ measured a diffusion barrier of $0.45 \mathrm{eV}$ with a preexponential factor of $4 \times 10^{12}$ $\mathrm{s}^{-1}$ with scanning electron microscopy. The values of the diffusion barriers calculated with molecular dynamics depend on the choice of potential: with the classical EAM potentials we find a barrier of $0.66 \mathrm{eV}$ (see above) whereas Sanders and DePristo ${ }^{60}$ find $0.42 \mathrm{eV}$ and $\nu_{0}=8 \times 10^{12} \mathrm{~s}^{-1}$ with a corrected effective medium method; Perkins and DePristo, ${ }^{52}$ using the same potentials but allowing the substrate to relax, find $0.5 \mathrm{eV}$ and $\nu_{0}=5 \times 10^{12} \mathrm{~s}^{-1}$.

We realize that the preexponential factor we obtain is very low. Actually we would expect it to be of the same order of magnitude as the vibrational frequency of the adatom (typically $10^{12}-10^{13} \mathrm{~s}^{-1}$ ), which could be understood as the attempt frequency for one hop. Small preexponential factors can be indications of complex diffusion mechanisms such as exchange, ${ }^{61,62}$ however, this particular mechanism seems to be excluded energetically by molecular dynamics calculations. ${ }^{52}$ It might also be that the limited range of temperatures in which we can measure the diffusion with helium is at the origin of an error on the preexponential factor. Actually in the literature preexponential factors vary in a large range (see, for example, Tsong ${ }^{3}$ and Bonzel ${ }^{4}$ ); the value we extracted from our data is in this range.

The result of the fits shows that the dimer has a much lower mobility than that of the monomer: we find that the hopping frequencies $\nu_{2}$ associated with the dimer are about 30 times smaller than the frequency $\nu_{1}$ associated with the monomer. However, this can be put into evidence only for 
the two highest temperatures ( 220 and $225 \mathrm{~K}$ ); for lower temperatures the mobility of the dimer is too low to be detected. The number of measured points is limited by the narrow temperature range where both diffusion characteristic times of the monomer and dimer coincide with the measurement characteristic times and the domain of validity of our model. This number is thus too low to get quantitatively the dynamical parameters for the diffusion of the dimer. It has, however, to be emphasized that the fits clearly indicate a lower mobility for the dimer than for the monomer: fits on the same data points by forcing $\nu_{2}=\nu_{1}$ and by leaving $\nu_{3}$ free reproduce only very badly the deposition curves. A similar difference between the diffusivity of atoms and dimers has been measured by Ehrlich ${ }^{9}$ in the case of Ir on $\operatorname{Ir}(111)$.

\section{Conclusions}

We have studied the dynamic of nucleation and growth of $\mathrm{Ag}$ on $\mathrm{Pd}(100)$ with TEAS. The reflected He beam intensity can be reproduced using a model coupling a rate equation approach and the geometrical overlap approach. This model allows one to extract the hopping frequencies of the monomer and dimer by fitting the theoretical curve to the attenuation of the specularly reflected $\mathrm{He}$ beam.

A diffusion barrier of $E_{d 1}=0.37 \pm 0.03 \mathrm{eV}$ with a preexponential factor of $\nu_{0}=8 \times 10^{9} \mathrm{~s}^{-1}$ is deduced by analyzing of the deposition curves at a substrate temperature varying between $T_{s}=180$ and $225 \mathrm{~K}$. The results are in fairly good agreement with different molecular dynamics simulations. The dimer seems to have a lower mobility than the monomer.

\section{ACKNOWLEDGMENTS}

We are grateful to Dr. C. Massobrio for providing us his molecular dynamics code as well as for sharing with us his expert knowledge in this field. This work was supported by the Swiss National Fund for Scientific Research (Grant No. 2000-040736.94).
${ }^{1}$ C. R. Henry, C. Chapon, C. Goyhenex, and R. Monot, Surf. Sci. 272, 283 (1992).

${ }^{2}$ X. P. Xu and D. W. Goodman, Catalysis Lett. 24, 31 (1994).

${ }^{3}$ T. T. Tsong, Rep. Prog. Phys 51, 759 (1988).

${ }^{4}$ H. P. Bonzel, in Diffusion in Metals and Alloys, edited by $\mathrm{H}$. Mehrer (Springer, Berlin, 1990), Chap. 13.

${ }^{5}$ A. G. Naumovets and Y. S. Vedula, Surf. Sci. Rep. 4, 365 (1985).

${ }^{6}$ R. Gomer, Rep. Prog. Phys. 53, 917 (1990).

${ }^{7}$ E. W. Müller and T. T. Tsong, Field Ion Microscopy, Principles and Applications (Elsevier, New York, 1969).

${ }^{8}$ N. Ernst and G. Ehrlich, Microscopic Methods in Metals, Topics in Current Physics Vol. 40 (Springer, Berlin, 1986).

${ }^{9}$ G. Ehrlich, Surf. Sci. 246, 1 (1991).

${ }^{10}$ G. W. Jones, J. M. Marcano, J. K. Nørskov, and J. A. Venables, Phys. Rev. Lett. 65, 3317 (1990).

${ }^{11}$ R. Jaklevic and L. Elie, Phys. Rev. Lett. 60, 120 (1988).

${ }^{12}$ H. Röder, E. Hahn, H. Brune, J. P. Bucher, and K. Kern, Nature 366, 141 (1993).

${ }^{13}$ Y. W. Mo, J. Kleiner, M. B. Webb, and M. G. Lagally, Phys. Rev. Lett. 66, 1998 (1991).

${ }^{14}$ S. C. Wang and G. Ehrlich, Phys. Rev. Lett. 71, 4174 (1993).

${ }^{15}$ W. F. Egelhoff and I. Jacob, Phys. Rev. Lett. 62, 921 (1989).

${ }^{16}$ D. E. Sanders and A. E. DePristo, Surf. Sci. 254, 341 (1991).

${ }^{17}$ G. De Lorenzi and G. Ehrlich, Surf. Sci. Lett. 293, L900 (1993).

${ }^{18}$ J. Frenken, J.-P. Toennies, and C. Woll, Phys. Rev. Lett. 60, 1727 (1988).

${ }^{19}$ H. Ernst, F. Fabre, and J. Lapujoulade, Phys. Rev. B 46, 1929 (1992).

${ }^{20}$ B. Poelsema, L. Verheij, and G. Comsa, Phys. Rev. Lett. 49, 1731 (1982).

${ }^{21}$ G. Rosenfeld, A. Becker, B. Poelsema, L. Verheij, and G. Comsa, Phys. Rev. Lett. 69, 917 (1992).

${ }^{22}$ J. M. Guglielmacci and M. Gillet, Thin Solid Films 68, 407 (1980).

${ }^{23}$ J. M. Guglielmacci and M. Gillet, Surf. Sci. 105, 386 (1981).

${ }^{24}$ J. M. Guglielmacci and M. Gillet, J. Cryst. Growth 57, 1558 (1982).
${ }^{25}$ K. Prince and V. Cháb, J. Electron. Spectros. Relat. Phenom. 52, 61 (1990).

${ }^{26}$ S. Chambers, S. Anderson, and J. Weaver, Phys. Rev. B 32, 4872 (1985).

${ }^{27}$ P. Pervan and M. Milun, Surf. Sci. 264, 135 (1992).

${ }^{28}$ R. Fischer, S. Schuppler, N. Fischer, T. Fauster, and W. Steinmann, Phys. Rev. Lett. 70, 654 (1993).

${ }^{29}$ B. Eisenhut, J. Stober, G. Rangelov, and T. Fauster, Phys. Rev. B 47, 12980 (1993).

${ }^{30}$ L. S. Perkins and A. E. DePristo, Surf. Sci. 319, 225 (1994).

${ }^{31}$ B. Poelsema and G. Comsa, Scattering of Thermal Energy Atoms from Disordered Surfaces, Springer Tracts in Modern Physics Vol. 115 (Springer-Verlag, Berlin, 1989).

${ }^{32}$ Note that the comparison of the experiment with Eq. (1) is a very sensitive test to check for random deposition.

${ }^{33}$ W. K. Burton, N. Cabrera, and F. C. Frank, Philos. Trans. R. Soc. London Ser. A 243, 299 (1951).

${ }^{34}$ J. Venables, Philos. Mag. 17, 697 (1973).

${ }^{35}$ B. Lewis and J. C. Anderson, Nucleation and Growth of Thin Films (Academic Press, London, 1978).

${ }^{36}$ J. A. Venables, Surf. Sci. 299/300, 798 (1994).

${ }^{37}$ G. Vandoni, C. Félix, R. Monot, J. Buttet, and W. Harbich, Surf. Sci. Lett. 320, L63 (1994).

${ }^{38}$ E. Hahn, Ph.D. thesis, EPF-Lausanne, 1994.

${ }^{39}$ Actually the capture zone depends also on the size of particle $i$ (i.e., the size of the cluster that is being captured), however, since this effect is small in comparison with the one described here, we neglegted it in the present paper.

${ }^{40}$ P. Erdös and S. J. Taylor, Acta Math. Acsd. Sci. Hung. 11, 137 (1960).

${ }^{41}$ E. W. Montroll and G. H. Weiss, J. Math. Phys. 6, 167 (1965).

${ }^{42}$ In the limit of zero island density, a better expression for the capture rate by the steps can be obtained by treating the problem with the diffusion equation, which can be integrated analytically in this case. This approach leads, however, to an underestimate of the capture rate by the steps when the island density on the terraces increases. 
${ }^{43}$ M. J. Stowell, Philos. Mag. 21, 125 (1970).

${ }^{44}$ B. Calpini, M. Croci, R. David, and R. Monot, J. Vac. Sci. Technol. A 10, 3221 (1992).

${ }^{45}$ M. J. Abrams and P. S. Weiss, Surf. Sci. 312, 1 (1994).

${ }^{46}$ R. Kunkel, B. Poelsema, L. K. Verheij, and G. Comsa, Phys. Rev. Lett. 65, 733 (1990).

${ }^{47}$ P. Stoltze and J. K. Norskov, Phys. Rev. B 48, 5607 (1993).

${ }^{48}$ S. C. Wang and G. Ehrlich, J. Chem. Phys. 94, 4071 (1991).

${ }^{49}$ P. Blandin and C. Massobrio, Surf. Sci. Lett. 279, L219 (1992).

${ }^{50}$ S. C. Wang and G. Ehrlich, Phys. Rev. Lett. 70, 41 (1993).

${ }^{51}$ S. M. Foiles, M. I. Baskes, and M. S. Daw, Phys. Rev. B 33, 7983 (1986).

${ }^{52}$ L. S. Perkins and A. E. DePristo, Surf. Sci. 319, 225 (1994), performed analogous calculations on the same substrate but using different potentials (Molecular dynamics/Monte Carlo corrected effective medium). They give a barrier of $0.5 \mathrm{eV}$, slightly lower than that of our calculation.

${ }^{53}$ C. L. Liu and J. B. Adams, Surf. Sci. 294, 197 (1993).
${ }^{54}$ P. J. Feibelman, Surf. Sci. Lett. 313, L801 (1994).

${ }^{55}$ G. Vandoni, C. Félix, and C. Massobrio, Phys. Rev. B 54, 1553 (1996).

${ }^{56}$ The analysis done by neglecting the corrections (no capture zones and no memory of the system) gives $\nu_{0}=3 \times 10^{-9} \mathrm{~s}^{-1}$ and $E_{d 1}=0.35 \mathrm{eV}$, while the same analysis taking into account only the correction of the capture zones leads to $\nu_{0}=9 \times 10^{-9} \mathrm{~s}^{-1}$ and $E_{d 1}=0.38 \mathrm{eV}$.

${ }^{57}$ J. Evans, D. Flynn-Sanders, and P. Thiel, Surf. Sci. 298, 378 (1993).

${ }^{58}$ K. Hartig, A. P. Janssen, and J. A. Venables, Surf. Sci. 74, 68 (1978).

${ }^{59}$ J. A. Venables, Phys. Rev. B 36, 4153 (1987).

${ }^{60}$ D. E. Sanders and A. E. DePristo, Surf. Sci. 260, 116 (1992).

${ }^{61}$ L. D. Roelofs, J. I. Martin, and R. Sheth, Surf. Sci. 250, 17 (1991).

${ }^{62}$ E. Ganz, S. K. Theiss, I.-S. Hwang, and J. Golovchenko, Phys. Rev. Lett. 68, 1567 (1992). 